\title{
Pediatric nasal epithelial cells are less permissive to SARS-CoV-2 replication compared to adult cells
}

Yanshan Zhu' ${ }^{1}$, Keng Yih Chew ${ }^{1}$, Anjana C. Karawita ${ }^{1}$, Ayaho Yamamoto ${ }^{2}$, Larisa L. Labzin ${ }^{3}$, Tejasri Yarlagadda ${ }^{4}$, Alexander A. Khromykh ${ }^{1,5}$, Claudia J. Stocks ${ }^{3}$, Yao Xia ${ }^{6}$, Tobias R. Kollmann ${ }^{7}$, David Martino ${ }^{7}$, Anthony Kicic ${ }^{7,8,9}$, Merja Joensuu ${ }^{10,11}$, Frédéric A. Meunier ${ }^{10,11,}$ Giuseppe Balistreri ${ }^{11,12}$ Helle Bielefeldt-Ohmann ${ }^{1,5,13}$, Asha C. Bowen ${ }^{7,13}$, Peter D. Sly ${ }^{2,14}$, Kirsten M. Spann ${ }^{4}$, Kirsty R. Short ${ }^{1,5^{*}}$

1 School of Chemistry and Molecular Biosciences, The University of Queensland, Brisbane, Australia

2 Child Health Research Centre, The University of Queensland, South Brisbane, QLD 4101, Australia

3 Institute for Molecular Bioscience, The University of Queensland, Brisbane, Australia 4 Centre for Immunology and Infection Control, Faculty of Health, School of Biomedical Sciences, Queensland University of Technology, Brisbane Queensland 4000, Australia 5 Australian Infectious Diseases Research Centre, Global Virus Network Centre of Excellence, Brisbane, Australia

6 School of Science, Edith Cowan University; School of Biomedical Science, The University of Western Australia, Perth, Australia

7 Wal-yan Respiratory Research Centre, Telethon Kids Institute, The University of Western Australia, Perth, Australia

8. Occupation and Environment, School of Public Health, Curtin University, Perth, Australia

9. Centre for Cell Therapy and Regenerative Medicine, School of Medicine and

Pharmacology, The University of Western Australia, Perth, Australia

10. Clem Jones Centre for Ageing Dementia Research, Queensland Brain Institute, The University of Queensland, Brisbane, QLD, Australia.

11. Queensland Brain Institute, The University of Queensland, Brisbane, QLD, Australia.

12 Department of Virology, Faculty of Medicine, University of Helsinki, Finland 13 School of Veterinary Science, The University of Queensland, Gatton, Australia 14 Department of Infectious Diseases, Perth Children's Hospital, Nedlands, Perth, Western Australia

\section{${ }^{*}$ Corresponding author:}

Kirsty R. Short, Ph.D., School of Chemistry and Molecular Biosciences, University of Queensland, Brisbane Australia

Phone: +61 7336 54226; Email: k.short@uq.edu.au 
51

52

53

54

\section{Abstract}

Children typically experience more mild symptoms of COVID-19 when compared to adults. There is a strong body of evidence that children are also be less susceptible to SARS-CoV-2 infection with the original Wuhan isolate. The reasons for reduced SARS-CoV-2 symptoms and infection in children remain unclear and may be influenced by a multitude of factors, including differences in target cell susceptibility and innate immune responses. Here, we use primary nasal epithelial cells from children and adults, differentiated at an air-liquid interface to show that SARS-CoV-2 (both the Wuhan isolate and the more recent Alpha variant) replicates to significantly lower titers in the nasal epithelial cells of children compared to those of adults. This was associated with a heightened antiviral response to SARS-CoV-2 in the nasal epithelial cells of children. Importantly, influenza virus, a virus whose transmission is frequently associated with pediatric infections, replicated in both adult and paediatric nasal epithelial cells to comparable titres. Taken together, these data show that the nasal epithelium of children supports lower infection and replication of SARS-CoV-2 than the adult nasal epithelium.

Keywords: SARS-CoV-2; pediatric nasal epithelial cells; anti-viral response; innate immune response; susceptibility to SARS-CoV-2 infection, alpha variant 


\section{INTRODUCTION}

Severe Acute Respiratory Syndrome-Coronavirus 2 (SARS-CoV-2), the causative agent of coronavirus disease-2019 (COVID-19), causes a broad range of clinical symptoms, ranging from asymptomatic infection to potentially fatal acute respiratory distress syndrome (ARDS). Children typically experience mild symptoms of COVID-19 when compared to adults ${ }^{1}$. There is also a significant body of evidence with the original Wuhan SARS-CoV-2 isolate that children are less susceptible to SARS-CoV-2 infection and less likely to transmit the virus. Specifically, a low rate of pediatric SARS-CoV-2 infections has been observed in multiple countries including China ${ }^{2}$, Italy ${ }^{3}$, the U.S.A ${ }^{4}$, Spain ${ }^{5}$ and Poland ${ }^{6}$. Similarly, in a metaanalysis of SARS-CoV-2 household transmission clusters early in the pandemic, children were significantly less likely to contract SARS-CoV-2 from infected family members compared to adult members of the household ${ }^{7}$. These findings have been echoed in multiple single site studies where, both within and outside of households, the infection rate of SARS-CoV-2 amongst children $<10$ years old is significantly lower than that of adults ${ }^{8}$. Reduced SARSCoV-2 infection and transmission is also observed in juvenile ferrets compared to their older counterparts ${ }^{9}$.

In the second year of the SARS-CoV-2 pandemic numerous viral variants have become prevalent, including the Alpha variant (B.1.1.7) which contains multiple mutations in the spike protein, the $\mathrm{N}$ protein and various open reading frames (ORFs) of the virus. The SARS-CoV-2 Alpha variant is of significant concern because of its increased transmissibility and possible increased virulence ${ }^{10}$. Early evidence suggests that the Alpha variant, similarly to original Wuhan isolate, is associated with low risk of severe disease in young children ${ }^{11,12}$. Some studies have suggested that children are more susceptible to the Alpha variant compared to the original Wuhan isolate ${ }^{13}$ whilst others have found little evidence of differential susceptibility ${ }^{14}$.

The reduced susceptibility of children to SARS-CoV-2 infection and disease (at least some instances) is in stark contrast to other seasonal respiratory viruses, such as influenza virus, where children are thought to play a major role in the spread of the virus ${ }^{15}$. The reasons for less frequent SARS-CoV-2 infection and symptoms in children, at least with the original Wuhan isolate, remain unclear and may be influenced by a multitude of factors. Pre-existing immunity to SARS-CoV-2 (likely derived from seasonal coronaviruses) may offer some form of crossprotection from infection in children ${ }^{16}$. Indeed, SARS-CoV-2 spike glycoprotein reactive antibodies in uninfected individuals are more prevalent amongst children and adolescents ${ }^{16}$.

It is also possible that nasal epithelial cells (NECs), the first site of infection, are fundamentally different in children compared to adults. Gene expression studies using the nasal epithelium of healthy individuals suggests that the transcript for the SARS-CoV-2 receptor, angiotensin 
converting enzyme-2 (ACE2), is expressed at lower levels in children compared to adults ${ }^{17}$. However, this has yet to be validated on a protein level. Moreover, this does not appear to be the case in all patient cohorts ${ }^{18,19}$. Following binding of the SARS-CoV-2 spike protein to ACE2, the host surface transmembrane serine protease 2 (TMPRSS2) is also involved in viral entry into the cell ${ }^{20}$. NECS from children express less TMPRSS 2 mRNA than those from adults, which may contribute to less frequent pediatric infections with SARS-CoV-2 ${ }^{21}$. However, this has also yet to be confirmed at protein level.

In addition to differential receptor expression, pediatric and adult NECs may also mount fundamentally different innate immune response to SARS-CoV-2. Recent RNA sequencing of the whole epithelium from pediatric and adult proximal airways suggests that there is a higher expression of genes associated with inflammation and the anti-viral response in children compared to adults 22,23 . Whilst increased inflammation and interferon production have previously been associated with elevated COVID-19 severity ${ }^{24}$, it is important to note that such studies refer to the inflammatory response in the lower respiratory tract, where any immunopathology may lead to respiratory distress ${ }^{25}$. In contrast, inflammation in the upper respiratory tract plays an important role in controlling early viral replication. Consistent with this supposition, nasopharyngeal swabs from SARS-CoV-2 infected children display elevated levels of interferons and inflammatory markers compared to those of SARS-CoV-2 infected adults ${ }^{18}$. However, whether this results in reduced replication of SARS-CoV-2 in the nasal epithelium of children remains to be determined.

Here, we use primary nasal epithelial cells (NECs), differentiated at an air-liquid interface, to investigate differential infection kinetics and antiviral responses to SARS-CoV-2 infection in children and adults. 


\section{METHODS}

173

174

175

176

177

178

179

180

181

182

183

184

185

186

187

188

189

190

191

192

193

194

195

196

197

198

199

200

201

202

203

204

205

206

207

208

209

210

\section{Cell collection and ethics statement}

Primary NECs were collected from healthy adult (aged 21 to 65 years old) donors by placing a sterile nasal mucosal curette (Arlington Scientific Inc., USA) in the mid-inferior portion of the inferior turbinate during June 2020 to May 2021. Informed consent was obtained from all donors. Primary NECs were obtained from healthy pediatric donors (aged 2 to 7 years old) in the same manner while under general anesthetic prior to elective surgery for sleep apnoea or tonsilitis in 2019. Children did not have any other unknown underlying condition. A total of 10 adult donors and 12 pediatric donors were used for this study. This study was approved bythe University of Queensland's Human Research Ethics Committee (2020001742), the Queensland Children's Hospital and Health Service Human Research Ethics Committee (HREC/16/QRCH/215) and Queensland University of Technology Human Research Ethics Committee 17000000039). Primary NECs were stored in freezing media (FBS with 10\% DMSO) after the second passage in culture.

\section{Cell culture}

African green monkey kidney epithelial Vero cells were maintained in MEM (Invitrogen), containing $10 \%(\mathrm{v} / \mathrm{v})$ heat-inactivated fetal bovine serum (Cytiva), $100 \mathrm{U} / \mathrm{ml}$ penicillin and streptomycin (Life Technologies Australia). Madin-Darby Canine Kidney (MDCK) cells were maintained in DMEM (Invitrogen), containing 10\% (v/v) heat-inactivated fetal bovine serum (Cytiva), $100 \mathrm{U} / \mathrm{ml}$ penicillin and streptomycin (Life Technologies Australia). All cell lines were obtained from American Type Culture Collection (ATCC; Virginia, USA). Primary NECs were expanded and passaged in Pneumacult EX Plus media (STEMCELL Technologies Inc, Canada). After initial expansion, NECs were seeded at a density of $4-5 \times 10^{5}$ cells/transwell on $6.5 \mathrm{~mm}$ transwell polyester membranes with 0.4 um pores (Corning Costar, USA) and cultured in EX Plus media (STEMCELL Technologies). Cells were monitored for confluence. When a confluent monolayer was achieved, cells were 'air-lifted' by removing the media from the apical chamber and replacing the basolateral media with Pneumacult air liquid interface (ALI) media (STEMCELL Technologies) ${ }^{26}$. Medium was replaced in the basal compartment three times a week, and the cells were maintained in ALI conditions for at least 3 weeks until ciliated cells and mucus were observed and cells obtained a transepithelial electrical resistance (TEER) measurement greater than $1000 \Omega$. Fully differentiated cultures were used in downstream infection experiments using influenza virus or SARS-CoV-2.

\section{Viral stocks}

SARS-CoV-2 isolate hCoV-19/Australia/QLD02/2020 (QLD02) (used as the original Wuhan isolate) and hCoV-19/Australia/QLD1517/2020(QLD1517) (GISAID accession EPI_ISL_944644; Alpha variant) were kindly provided by Queensland Health Forensic \& Scientific Services, Queensland Department of Health. Virus was amplified in Vero cells 
expressing human TMPRSS2 and titrated by plaque assay ${ }^{27}$. All studies with SARS-CoV-2 were performed under physical containment 3 (PC3) conditions and were approved by the University of Queensland Biosafety Committee

(IBC/374B/SCMB/2020). A/Auckland/4/2009(H1N1) (Auckland/09) stocks were prepared in embryonated chicken eggs as previously described ${ }^{28}$. Viral titers were determined by plaque assays on MDCK cells, as previously described ${ }^{29}$.

\section{Viral infection}

Differentiate adult and pediatric NECs were infected with mock (PBS), QLD02 (1.25 x $10^{5}$ PFU), QLD1517 (2.4 x 104 PFU) or Auckland/09 (1.25 x 10 5 PFU). Specifically, 100uL of virus or PBS was placed on the epithelial surface in the apical compartment and incubated for 1 hour at $37^{\circ} \mathrm{C}$. Following incubation, excess virus was removed from the transwell and cells were incubated at $37^{\circ} \mathrm{C}$ with $5 \% \mathrm{CO}_{2}$. Every 24 hours the basolateral media was refreshed with $1 \mathrm{~mL}$ of new ALI media. At pre-determined timepoints post-infection $100 \mu \mathrm{L}$ of PBS (or in the case of influenza virus PBS $+0.1 \mu \mathrm{g}$ of TPCK-treated trypsin (Worthington, USA)) was added to the apical compartment and cells were incubated at $37^{\circ} \mathrm{C}$ with $5 \% \mathrm{CO}_{2}$ for 10 minutes. The apical supernatant was subsequently removed and stored at $-80^{\circ} \mathrm{C}$. Cells were lysed with Buffer RLT (Qiagen, USA) containing $0.01 \% \beta$-mercaptoethanol for RNA analysis. Alternatively, cells were lysed in 2\% SDS/PBS lysis buffer (2\% SDS/PBS buffer, 10\% 10x PhosSTOP, 4\% $25 \mathrm{x}$ protease inhibitor) for protein analysis or fixed overnight in $4 \%$ paraformaldehyde for histology.

\section{Histology}

Fixed cells on a transwell membrane were routine processed and embedded in paraffin, sectioned at $5 \mu \mathrm{m}$ and subsequently stained with hematoxylin and eosin (H\&E) or Periodic acidSchiff (PAS). Sections were assessed for cellular morphology by a veterinary pathologist (H.B.O.) blinded to the experimental design.

\section{Immunofluorescence}

Differentiated epithelial cells grown on a transwell membrane were fixed with $4 \%$ paraformaldehyde (Cat\#15710, Electron Microscopy Sciences) in PBS for 45 minutes at room temperature, followed by a blocking with $0.5 \%$ BSA (Sigma) in PBS for 30 minutes and permeabilization with $0.02 \%$ of Triton X-100 (Sigma) in PBS for $15 \mathrm{~min}$ at room temperature. After washing twice with PBS/BSA and a second blocking step for $10 \mathrm{~min}$ at room temperature, samples were incubated with primary antibodies overnight at $4^{\circ} \mathrm{C}$. Primary antibodies were diluted in 0.5\% BSA in PBS blocking solution: 1:400 ZO-1 (Cat\#40-2200, Thermo Fisher Scientific); 1:1000 MUC5AC (Cat\#MA5-12178, ThermoFisher Scientific); 1:500 ACE2 (Cat\#AF933, R\&D Systems). After three washing steps with $0.5 \%$ BSA/PBS for 5 minutes each time, the samples were incubated in secondary antibody: 1:1000 Alexa Flour 555 donkey 
anti-goat (Cat\#A21432, Invitrogen) for 2.5 hours at room temperature in dark, and after three washes in PBS and three washes with $0.5 \%$ BSA/PBS, the cells were incubated with a 1:1000 Alexa Fluor 647 goat anti-mouse (Cat\#A32728, Invitrogen) for 2.5 hours at room temperature covered from light. The cells were simultaneously stained with 1:400 Alexa Fluor 647 Phalloidin (Cat\#A22287 Invitrogen) and 1:1000 DAPI. After three washes in PBS, the transwell membranes with cells were cut with a scalpel, briefly dipped in milli-q water, and mounted on a class slide using ProLong Gold Antifade Mountant (Cat\# P10144, ThermoFisher Scientific). Mounted samples were imaged on a spinning-disk confocal system (Marianas; 3I, Inc.) consisting of a Axio Observer Z1 (Carl Zeiss) equipped with a CSU-W1 spinning-disk head (Yokogawa Corporation of America), ORCA-Flash4.0 v2 sCMOS camera (Hamamatsu Photonics), and 63x 1.4 NA / Plan-Apochromat / $180 \mu \mathrm{m}$ WD objective. Image acquisition was performed using SlideBook 6.0 (3I, Inc). 150 optical sections from five random regions of interest (ROIs) from each sample were acquired from the top of the differentiated epithelial cells. Image processing was performed using Fiji/ImageJ (Version 2.1.0/1.53c) as follows: Background was reduced using the Substract Backgound 50 pixel rolling ball radius, and the mean fluorescence intensity (MFI, a.u. arbitrary units) was measured from the average intensity images.

\section{Western Blot}

For total cell lysates, cells were washed twice with cold PBS and lysed with 2\% SDS/PBS lysis buffer ( $2 \%$ SDS/PBS buffer, 10\% 10x PhosSTOP, 4\% 25x protease inhibitor). Pierce BCA protein assay kit (Thermo Fisher Scientific) was used to equalize protein amounts and SDSsample buffer containing $100 \mathrm{mM}$ DTT (Astral Scientific) was added. Samples were boiled at $100^{\circ} \mathrm{C}$ for 10 minutes to denature proteins. Proteins were separated on $4-15 \%$ mini protean TGX precast gels (Biorad) in running buffer (200 mM Glycine, $25 \mathrm{mM}$ Tris, 0.1\% SDS, pH8.6), transferred to nitrocellulose membrane (Cat\#1620112, BioRad) in blot buffer (48 nM Tris, 39 nM Glycine, $0.04 \%$ SDS, 20\% MeOH) and subsequently blocked with 5\% (w/v) BSA in Trisbuffered saline with Tween 20 (TBST) for 30 minutes. The immunoblots were analyzed using primary antibodies incubated overnight at $4{ }^{\circ} \mathrm{C}$ and secondary antibodies linked to horseradish peroxidase (HRP) (Invitrogen), and after each step immunoblots were washed 4x with TBST. HRP signals were visualized by enhanced chemiluminescence (ECL) (BioRad) and imaged with a Chemidoc (BioRad). Primary antibodies include GAPDH (14C10) Rabbit monoclonal antibody (1:2500 dilution, Cat\#2118, Cell Signaling Technology), rabbit anti-SARS-CoV-2 Nucleoprotein/NP antibody (1:1000 dilution, Cat\#40143-R040, Sino Biological), goat polyclonal ACE2 (1:500 dilution, Cat\#AF933, R\&D Systems), rabbit anti-TMPRSS2 antibody (1:1000 dilution, Cat\#ab109131, Abcam). ImageJ was used to quantify the protein expression level relative to GAPDH levels. 


\section{Quantification of infectious virus}

SARS-CoV-2 titers in cell culture supernatants were determined by plaque assay on Vero cells, as described previously ${ }^{27}$. Influenza virus titers in cell culture supernatants were determined by plaque assay on MDCK cells ${ }^{29}$.

\section{RNA extraction and quantitative Reverse Transcription PCR (qRT-PCR)}

RNA was extracted from NECs using Nucleozole reagent according to the manufacturer's instructions, DNA was removed by DNase I (Thermo Fisher Scientific) treatment and $1 \mu \mathrm{g}$ DNA-free RNA was reverse transcribed into cDNA using the High Capacity cDNA Reverse Transcription Kit (Applied Biosystems) on a Mastercycler Thermocycler (Eppendorf, Hamburg, Germany) according to the manufacturer's instructions using random primers. Real-time PCR was performed on generated cDNA with SYBER Green (Invitrogen) using QuantStudio 6 Flex Real-Time PCR System, an Applied Biosystems Real-Time PCR Instruments (Thermo Fisher Scientific). Gene expression was normalized relative to glyceraldehyde 3-phosphate dehydrogenase (GAPDH) expression, fold change was calculated using the $\Delta \Delta \mathrm{Ct}$ method. All primers used in this study are listed in Table 1.

Table 1. Primers used in the present study

\begin{tabular}{cl}
\hline Primer name & Sequence (5'-3') \\
\hline $\mathrm{h} I F I T 1$ & FW:TTGCCTGGATGTATTACCAC \\
& RV: GCTTCTTGCAAATGTTCTCC \\
$\mathrm{h} C X C L 10$ & FW: GTGGCATTCAAGGAGTACCTC \\
& RV: GCCTTCGATTCTGGATTCAGACA \\
$\mathrm{h} I S G 15$ & FW: GAGAGGCAGCGAACTCATCT; \\
& RV: CTTCAGCTCTGACACCGACA) \\
$\mathrm{h} G A P D H$ & FW: CGAGATCCCTCCAAAATCAA; \\
& RV: TTCACACCCATGACGAACAT \\
\hline
\end{tabular}

\section{RNA Sequencing}

RNA-Seq libraries were prepared using the Illumina stranded total RNA prep ligation with the Ribo-Zero plus kit (Illumina) and IDT for Illumina RNA UD Indexes according to the standard manufacturer's protocol. Briefly, 50ng of total RNA was depleted of rRNA and then fragmented by heat. cDNA was synthesized from the fragmented RNA using random primers. The first strand cDNA was converted into dsDNA in the presence of dUTP to maintain the 'strandedness' of the library. The 3' ends of the cDNA were adenylated and pre-index anchors were ligated. The libraries were then amplified with 14-16 cycles of PCR incorporating unique indexes for each sample to produce libraries ready for sequencing. The libraries were quantified on the Perkin Elmer LabChip GX Touch with the DNA High Sensitivity Reagent kit (Perkin Elmer). Libraries were pooled in equimolar ratios, and the pool was quantified by qPCR using 
the KAPA Library Quantification Kit - illumina/Universal (KAPA Biosystems) in combination with the Life Technologies Viia 7 real time PCR instrument.

Sequencing was performed using the Illumina NextSeq500 (NextSeq control software v2.2.0 / Real Time Analysis v2.4.11). The library pool was diluted and denatured according to the standard NextSeq protocol and sequenced to generate single-end 76 bp reads using a 75 cycle NextSeq500/550 High Output reagent Kit v2.5 (Illumina). After sequencing, fastq files were generated using bcl2fastq2 (v2.20.0.422), which included trimming the first cycle of the insert read. Library preparation and sequencing was performed at the Institute for Molecular Bioscience Sequencing Facility (University of Queensland).

\section{RNA Sequencing analysis}

The quality of the trimmed RNA-seq reads was assessed with FastQC ${ }^{30}$ and MultiQC ${ }^{31}$. Salmon $^{32}$ was used for transcript quantification from human transcriptome (GENCODE Release 36, accessed in December 2020). A decoy aware transcriptome file was created for Salmon transcript quantification followed by the transcriptome index ${ }^{32}$. The $\mathrm{R}$ package, DESeq $2^{33}$ was then used for differential gene expression (DGE) analysis and further validated through using the limma $\mathrm{R}$ package ${ }^{34}$ with Voom transformation ${ }^{35}$. DGEs between virus and mock infected samples were analyzed by controlling the effect of the age group and gender of the individual samples, genes with adjusted $p$-value less than 0.05 were considered significant. Gene set enrichment analysis was performed using the R package GOseq ${ }^{36}$. All the $\mathrm{R}$ scripts were run on R-Studio platform ( RStudio Team 2020, v 1.4.1717).

\section{Code and data availability}

RNA-seq data is deposited at European Nucleotide Archive under the project- PRJEB43102. The scripts used for RNA-seq data analysis including differential gene expression and gene set enrichment analysis can be found in https://github.com/akaraw/Yanshan_Zhu_et_al.

\section{Statistical analysis}

Where sufficient cell numbers were present, samples were performed in duplicate, and the results were averaged and shown as a single data point. If sufficient cells were not present, a single transwell was used to determine the response of that donor to viral infection. Data were tested for normality using the Shapiro-Wilk test. Outliers of continual variables were removed using ROUT's test $(\mathrm{Q}=1 \%)$. Where data were normally distributed, data was analyzed using an unpaired two-tailed student's t-test. Where data were not normally distributed, data was analyzed using a Mann-Whitney U test. Significance was set at $p<0.05$. 


\section{RESULTS}

\section{Pediatric nasal epithelial cells are phenotypically different to adult nasal epithelial cells}

To investigate the role of NECs in SARS-CoV-2 infection, adult and pediatric NECs were differentiated at an air-liquid interface. The phenotype of these cells at baseline (i.e., prior to infection) was then assessed. Adult NECs grew as a pseudostratified columnar epithelium with scattered goblet cells and ciliated epithelial cells (Figure 1A). Pediatric NECs also grew as a pseudostratified columnar epithelium with ciliated epithelial cells and goblet cells (Figure 1A). However, scattered cells with pyknotic nucleus and condensed cytoplasm were also observed, leaving pseudocysts in the epithelium (Supplementary Figure 1). This is potentially indicative of higher cell turn-over and metabolic rate in the pediatric epithelial cells ${ }^{37,38}$. Immunofluorescence images of zonal occludens-1 (ZO-1) stained NECs show that tight junction proteins were built up closely towards the apical region of both adult and pediatric cells (Figure 1B). PAS staining indicated the presence of mucus producing cells (Figure 1A) in both pediatric and adult NECs. Consistent with these data, MUC5AC staining was detected exclusively on the apical layer, thus demonstrating mucus secretion by differentiated NECs (Figure 1B).

Previous mRNA expression studies suggest that pediatric NECs express lower levels of $A C E 2$ and TMPRSS2 compared to their adult counterparts ${ }^{17,21}$. However, these findings are inconsistent between patient cohorts and have not been investigated at a protein level ${ }^{19}$. Immunofluorescence staining suggested that pediatric NECs had lower surface levels of ACE2 compared to their adult counterparts (Figure 1B) although a limited sample size precluded statistical analysis (Figure 1C). Accordingly, we sought to confirm these data using western blot on the NECs from a larger number of donors $(n=5)$ (Figure 1D). Whilst the same trend was observed by western blot (increased levels of ACE2 in adult NECs) this failed to reach statistical significance (Figure 1D). There was no observable trend in TMPRSS2 levels between adult and pediatric NECs (Figure 1D).

\section{Pediatric nasal epithelial cells are less permissive to $\mathrm{SARS}-\mathrm{CoV}-2$ replication}

We next sought to determine if pediatric NECs were less susceptible than adult NECs to SARSCOV-2 (QLD02) replication. Strikingly, significantly reduced SARS-CoV-2 replication was observed in pediatric NECs at 24- and 72-hours post-infection (h.p.i) (Figure 2A). Reduced SARS-CoV-2 N protein level was also observed in pediatric NECs at 72 h.p.i, although this did not reach statistical significance $(p=0.07$; Figure $2 B$ and $C$, Supplementary Figure 2). To determine if decreased viral replication was specific to SARS-CoV-2, these experiments were repeated using influenza A virus, which is one of the many respiratory viruses known to be highly transmissible amongst children ${ }^{39}$. No significant difference in influenza A virus replication in pediatric NECs compared to adult cells was observed at 24-, 48- and 72-hour post-infection (Figure 2D). 


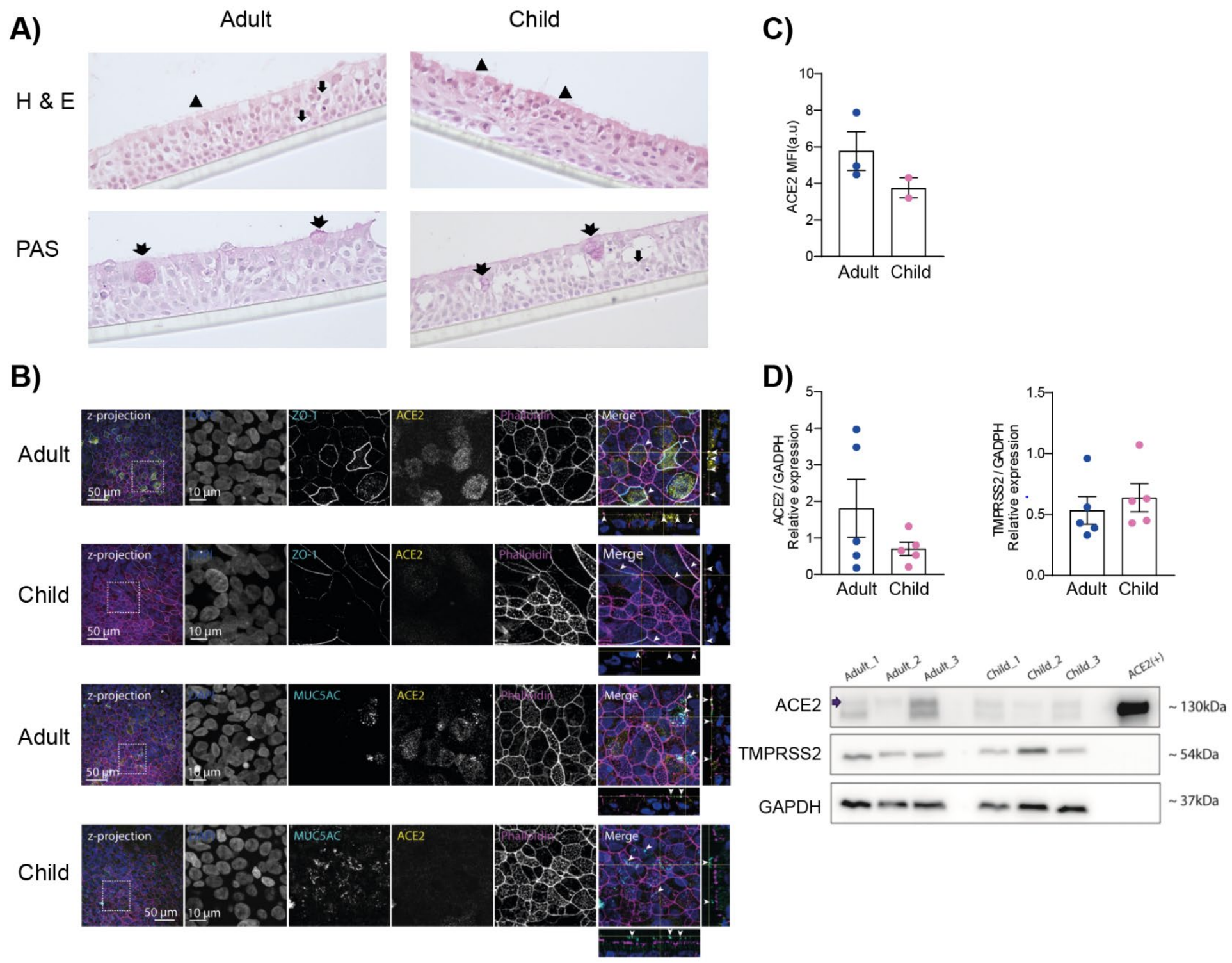

Figure 1. Pediatric nasal epithelial cells are phenotypically different to adult nasal epithelial cells. A) Representative H\&E and PAS-stained sections of pediatric and adult NECs culture differentiated at an air-liquid interface (representative of 2 adult ( 1 female, 1 male) and 3 pediatric donors ( 1 female, 2 males)). Arrowheads indicate ciliated cells, arrows indicate goblet cells and double-tailed arrows indicate mucus producing cells as determined by PAS staining. Images taken at 400x magnification. B) Representative z-projections (150 optical sections) of pediatric and adult NECs cultures differentiated at an air-liquid interface and immunolabelled against endogenous ZO-1 and ACE2 (cyan and yellow, respectively, top panels), and MUC5AC and ACE2 (cyan and yellow, respectively, bottom panels). Cells were also stained with DAPI (blue) and phalloidin (magenta) to indicate the nucleus and actin

408 filaments, respectively. The area in the dotted box in the images on left are shown magnified in 409 the respective rows $(10 \mu \mathrm{m}$ bar applies to all images in the row). The merged image on right 410 shows the orthogonal view of the z-stacks. The arrowheads indicate the zonal occludens-1 (ZO1) stained profiles (top panels) and mucus secretion (MUC5AC) in the lower panels. C) 412 Quantification of ACE2 immunofluorescence as described in the Materials and Methods. Mean $413 \pm$ SEM is shown. Each data point represents the average of five separate images taken from one 
donor (pediatric ( 1 female, 1 male) and adult ( 2 females, 1 male) donor). D) Relative ACE2 and TMPRSS2 protein levels compared to GAPDH in pediatric ( 3 females, 2 males) and adult (4 females, 1 male) NECs. Each data point represents a different donor. Mean \pm SEM is shown. C) Representative western blot of NECs from three adult and three pediatric donors blotted for ACE2, TMPRSS2 and GAPDH. ACE2 is indicated with an arrow.

A)

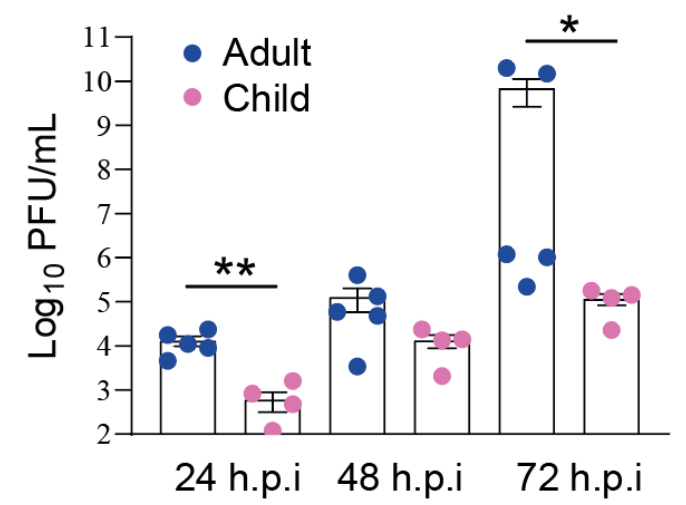

C)

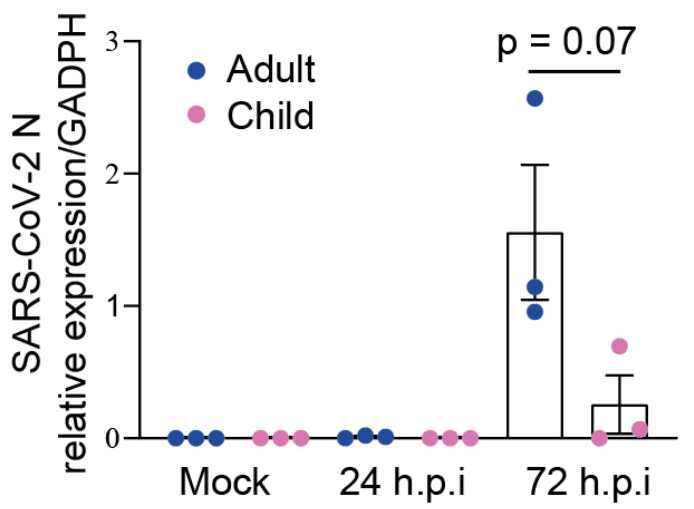

B)

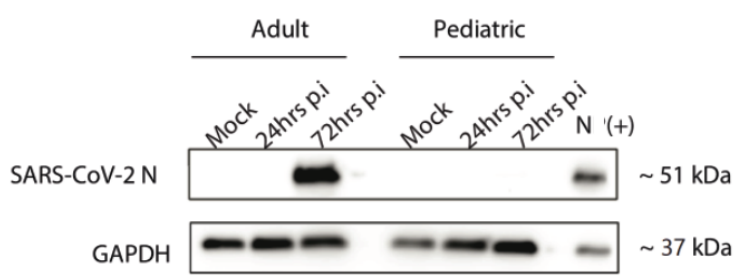

D)

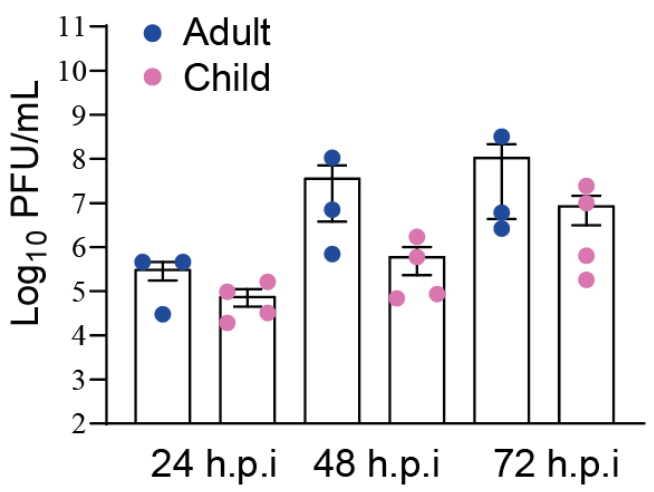

Figure 2. Lower replication of SARS-CoV-2 in pediatric nasal epithelial cells. A) Plaque forming units (PFU) of SARS-CoV-2 (QLD02) from the apical surface of nasal epithelial cells NECs obtained at 24, 48 and 72 h.p.i. B) Representative western blot of adult and pediatric donor blotted for SARS-CoV-2 N at various timepoints post-infection. All the western blot results ( $n=3$ adults and $n=3$ children) for SARS-CoV-2 N are shown as Supplementary Figure 2. C) Relative SARS-CoV-2 N levels compared to GAPDH in pediatric and adult NECs. D) Plaque forming units (PFU) of influenza A virus from the apical surface of NECs at various timepoints post-infection. Each data point represents a separate donor. Mean \pm SEM is shown. $p<0.05^{*}, p<0.01^{* *}$. Statistical analysis performed as described in the Materials and Methods. 


\section{Pediatric nasal epithelial cells mount a strong anti-viral response to SARS-CoV-2}

To gain a further insight into the observed decrease of SARS-CoV-2 replication in pediatric NECs RNA Seq was performed on infected adult and pediatric cells 72 hours post-SARS-CoV2 (QLD02) infection. PCA analysis showed that infected cells formed distinct clusters depending on whether they were derived from pediatric or adult donors (Figure 3A). Numerous differentially genes were recorded in infected cells (Figure 3B). In infected pediatric NECs, gene ontology (GO) enrichment analysis (Figure 3C) demonstrated a strong interferon response, with GO terms such as 'viral process', 'type I interferon signaling', 'response to virus', 'regulation of defense response to virus', 'negative regulation of viral genome replication', 'defense response to virus' and 'cellular response to interferon alpha'. None of these GO terms were identified amongst the top differentially expressed GO terms in adult cells infected with SARS-CoV-2 (Figure 3D). In contrast, GO terms such as 'cellular response to sterol', 'Wnt signalling pathway' and 'response to tumor necrosis factor' were recorded. To confirm that these data were not restricted to a DESeq2 analysis, gene expression data were also analyzed using limma (Table $2 \& 3$ ). Once again, in infected pediatric NECs GO terms such as 'response to virus', 'cellular response to cytokine stimulus' and 'defense response to virus' were recorded (Table 2). In contrast, infected adult NECs were associated with GO terms such as 'detection of stimulus involved in sensory perception' and 'sensory perception' (Table 3). To further validate these data, we assessed gene expression by qPCR of three genes associated with inflammatory/anti-viral response - interferon-induced protein with tetratricopeptide repeats 1 (IFIT1); C-X-C motif chemokine ligand 10 (CXCL10) and interferon stimulated gene 15 (ISG15). Consistent with our RNA Seq data, infected pediatric NECs had significantly higher levels of IFIT1 and ISG15 compared to infected adult NECs (Figure 4A). Interestingly, these data were not restricted to SARS-CoV-2 infection and a similar expression profile was observed following influenza A virus infection (Figure 4B).

It has been suggested that children may be more susceptible to the recent SARS-CoV-2 variants of concern (VOC) compared to the original SARS-CoV-2 isolate. To determine if our findings were restricted to the parental Wuhan isolate, we infected pediatric and adult NECs with the Alpha variant (QLD1517). Consistent with our previous data we observed increased viral replication in adult NECs compared to those derived from pediatric donors (Figure 5A). This differential replication was associated with a differential expression of key interferon associated genes (Figure 5B). 
bioRxiv preprint doi: https://doi.org/10.1101/2021.03.08.434300; this version posted August 24, 2021. The copyright holder for this preprint (which was not certified by peer review) is the author/funder. All rights reserved. No reuse allowed without permission.
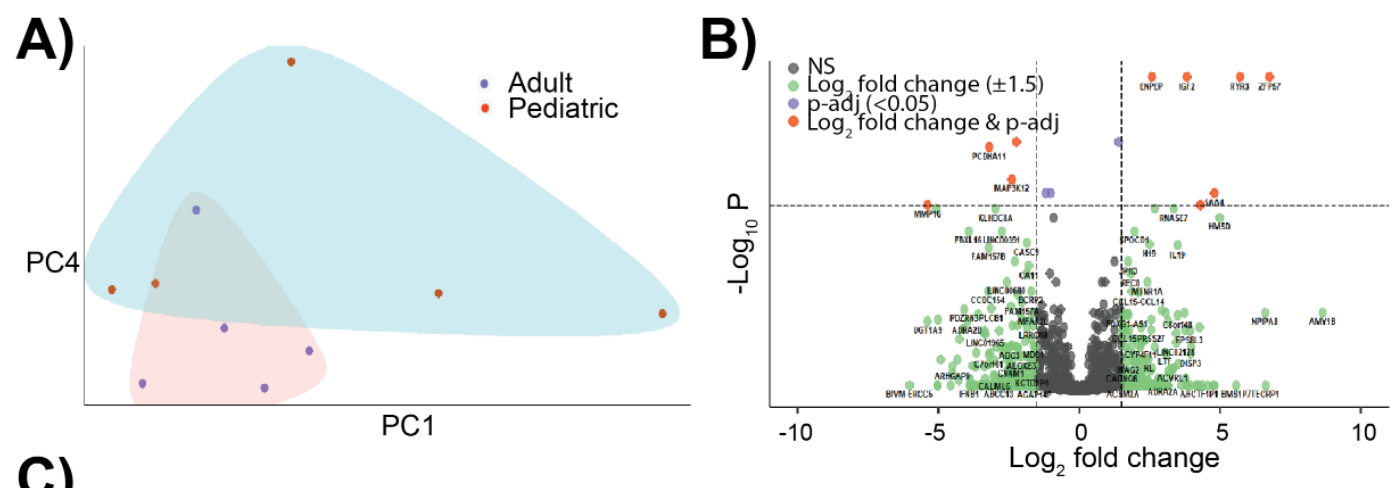

C)

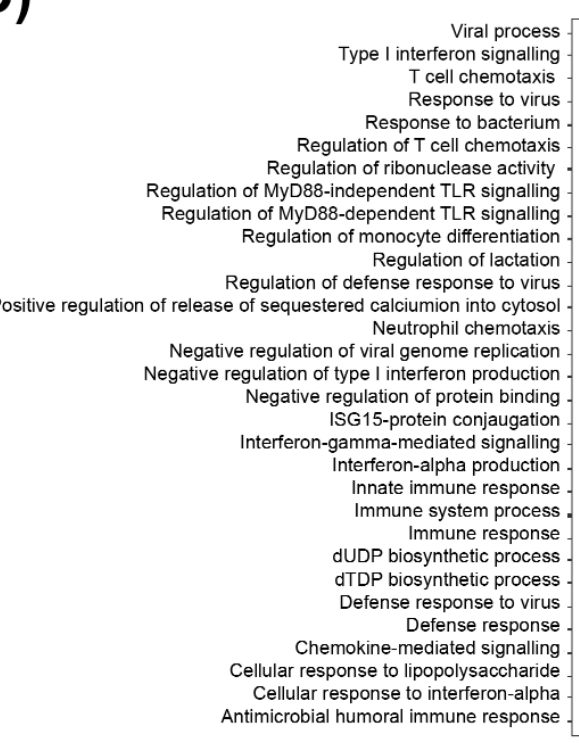

D)

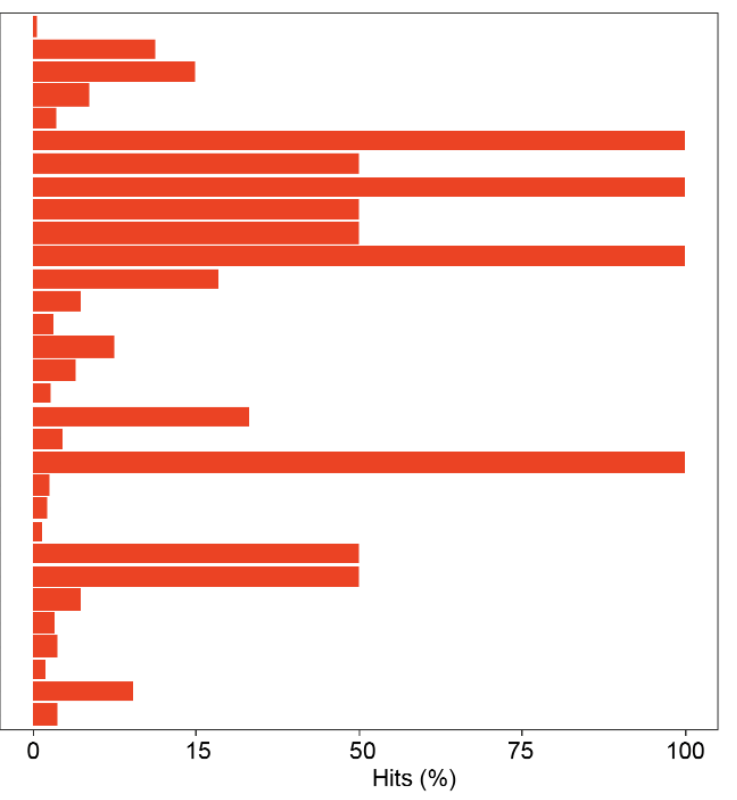

Normalised GO Enrichment Score of pediatric samples

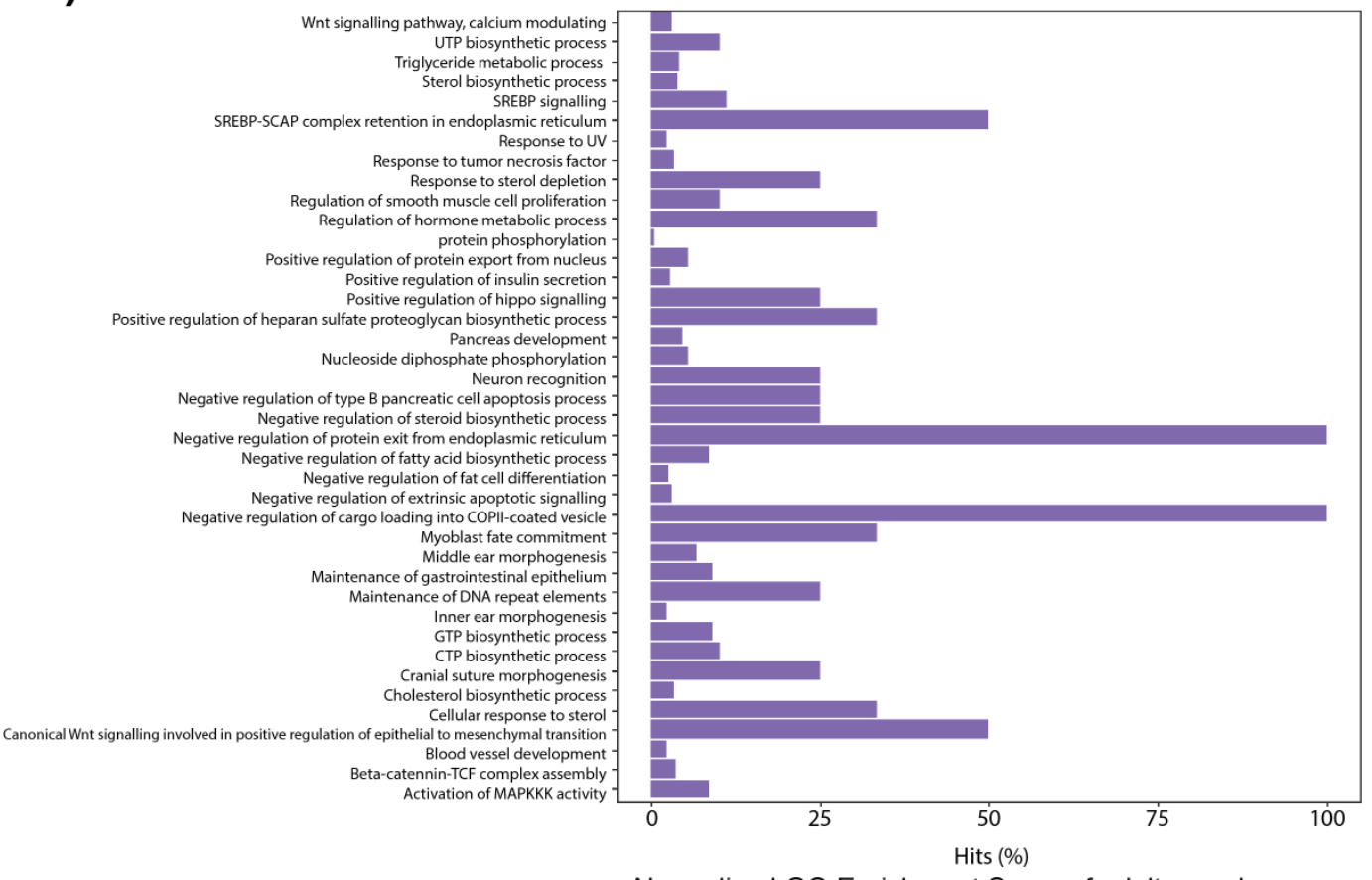

Normalised GO Enrichment Score of adult samples 
Figure 3: Pediatric epithelial cells have a different transcriptional response to SARS-CoV2. A) Principal component analysis for the global transcriptional response of naive pediatric and adult NECs. Data points represent individual donors. B) Volcano plot illustrating differentially expressed genes (DEGs) of naive pediatric NECs compared to adult cells. DEGs statistically different between the two patient groups with a fold change of $>1.5$ are indicated in orange. DEGs statistically different between two groups with a fold change of $<1.5$ are shown in purple. DEGs not statistically different between two groups with a fold change of $>1.5$ are shown in green. NS = not significant. C) Gene ontology (GO) analysis of DEGs in infected pediatric NECs were displayed by the bar chart. The bars of significantly GO enriched (Overrepresented $p$ value $<0.05$ ) results were marked in red, $x$-axis reflects the gene count hits as a percentage over genes in each GO category; y-axis reflects different GO terms. D) Gene ontology (GO) analysis of DEGs in adult NECs were displayed by the bar chart. The bars of significantly enriched GO (Overrepresented $p$ value $<0.05)$ enrichment results were marked in purple and represents the gene count hits (as a percentage over number of genes in a given category); y-axis reflects different GO terms.

Table 2: Significantly enriched gene ontology (Overrepresented $p$ value $<0.05$ ) terms in SARS-CoV-2 infected pediatric cells (relative to naïve cells)

\begin{tabular}{cc}
\hline & Term \\
\cline { 2 - 2 } GO:0071345 & cellular response to cytokine stimulus \\
GO:0019221 & cytokine-mediated signaling pathway \\
GO:0007166 & cell surface receptor signaling pathway \\
GO:0009615 & response to virus \\
GO:0007165 & signal transduction \\
GO:0034097 & response to cytokine \\
GO:0051607 & defense response to virus \\
GO:0008219 & cell death \\
GO:0012501 & programmed cell death \\
GO:0065008 & regulation of biological quality \\
\hline
\end{tabular}


Table 3: Significantly enriched gene ontology (Overrepresented $p$ value $<0.05$ ) terms in SARS-CoV-2 infected adult cells (relative to naïve cells)

506

\begin{tabular}{|c|c|}
\hline & Term \\
\hline GO:0050907 & Detection of chemical stimulus involved in sensory perception \\
\hline GO:0050906 & detection of stimulus involved in sensory perception \\
\hline GO:0009593 & detection of chemical stimulus \\
\hline GO:0007606 & sensory perception of chemical stimulus \\
\hline GO:0051606 & detection of stimulus \\
\hline GO:0050911 & detection of chemical stimulus involved in sensory perception of smell \\
\hline GO:0007186 & G protein-coupled receptor signaling pathway \\
\hline GO:0007608 & Sensory perception of smell \\
\hline GO:0007600 & sensory perception \\
\hline GO:0003008 & system process \\
\hline GO:0050877 & nervous system process \\
\hline GO:0002027 & regulation of heart rate \\
\hline GO:0003015 & heart process \\
\hline GO:0060047 & heart contraction \\
\hline GO:0008016 & regulation of heart contraction \\
\hline GO:0006805 & xenobiotic metabolic process \\
\hline GO:0001580 & $\begin{array}{l}\text { detection of chemical stimulus involved in sensory perception of bitter } \\
\text { taste }\end{array}$ \\
\hline GO:0050912 & detection of chemical stimulus involved in sensory perception of taste \\
\hline GO:0050913 & sensory perception of bitter taste \\
\hline GO:0007187 & G protein-coupled receptor signaling \\
\hline
\end{tabular}


A)
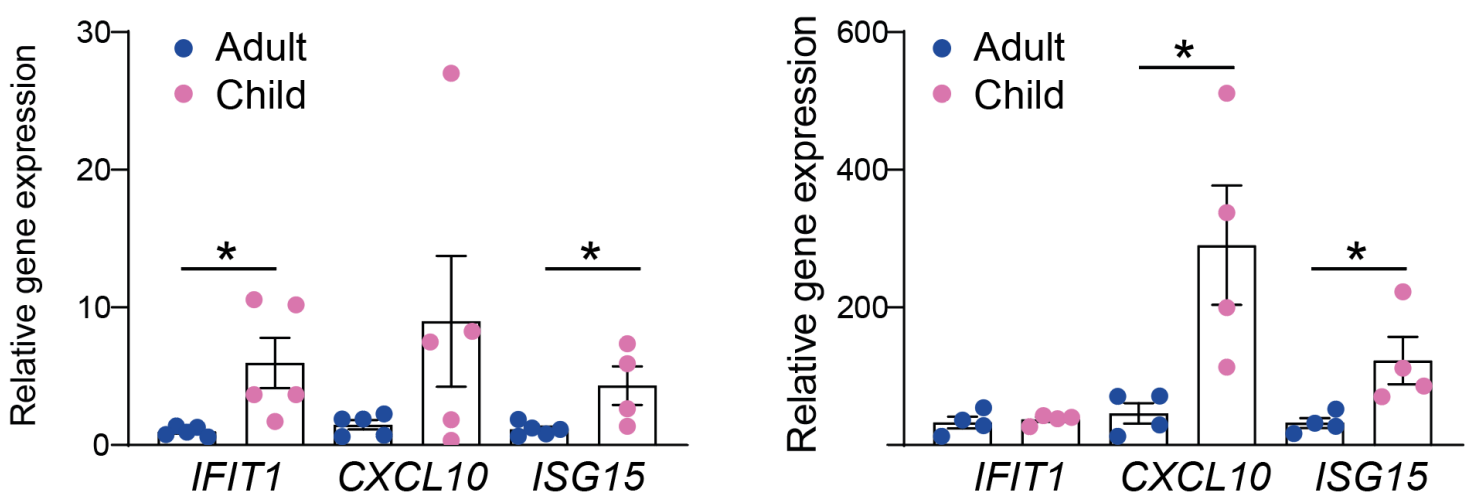

B)

Figure 4: Pediatric epithelial cells have a stronger type I IFN response to viral infection. A) Expression of interferon associated genes in SARS-CoV-2 (QLD02) infected epithelial cells relative to uninfected controls (48 hours post-infection). B) Expression of interferon associated genes in influenza A virus infected epithelial cells relative to uninfected controls (48 hours post-infection). Each data point represents a different donor. Gene expression (fold change) was calculated using the $\Delta \Delta \mathrm{Ct}$ method relative to GAPDH expression. Mean $\pm \mathrm{SEM}$ is shown. $p<0.05^{*}$, statistical analysis performed as described in the Materials and Methods.

A)

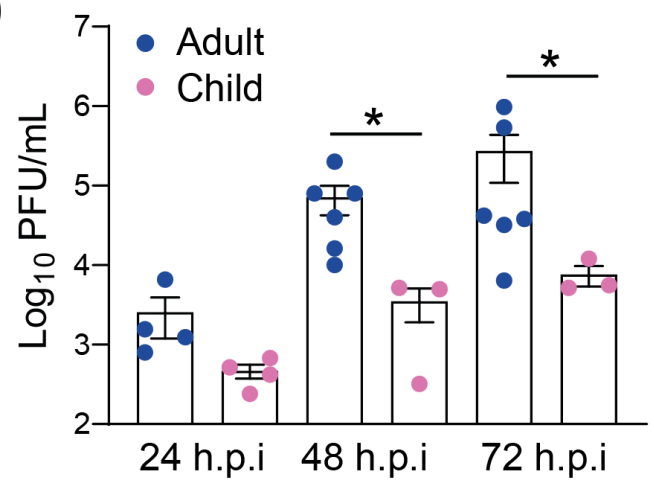

B)

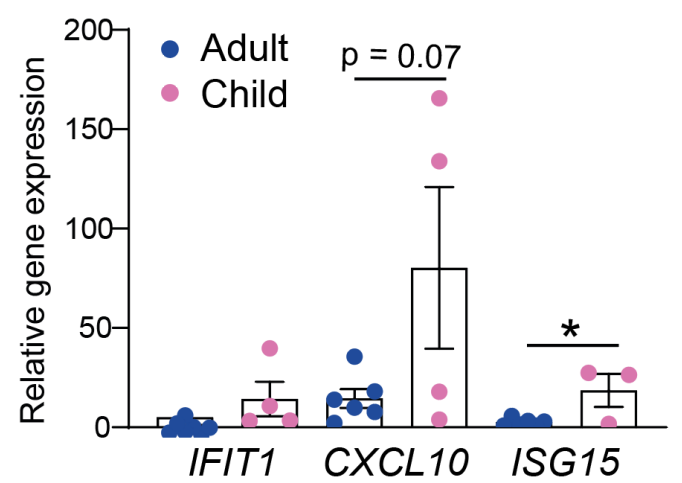

Figure 5. Lower replication of SARS-CoV-2 Alpha variant in pediatric nasal epithelial cells. A) Plaque forming units (PFU) of SARS-CoV-2 from the apical surface of nasal epithelial cells NECs obtained at 24, 48 and 72 h.p.i. B) Expression of interferon associated genes in influenza A virus infected epithelial cells relative to uninfected controls (48 h.p.i). Each data point represents a different donor. Gene expression (fold change) was calculated using the $\Delta \Delta \mathrm{Ct}$ method relative to GAPDH expression. Mean \pm SEM is shown. $p<0.05^{*}$. Statistical analysis performed as described in the Materials and Methods. 
543

544

545

546

547

548

549

550

551

552

553

554

555

556

557

558

559

560

561

562

563

564

565

566

567

568

569

570

571

572

573

574

575

576

577

578

579

580

581

\section{DISCUSSION}

Large clinical data sets and systematic reviews suggest that children are less often infected and symptomatic with SARS-CoV-2 than adults ${ }^{7,40-42}$. However, the mechanisms driving these observations have been unclear. Here, we have provided the first experimental evidence that the pediatric nasal epithelium may play an important role in reducing the susceptibility of children to SARS-CoV-2 infection.

Previous studies have suggested that the reduced susceptibility of children to SARS-CoV-2 infection is due to reduced expression of ACE2 and TMPRSS2 mRNA. Specifically, it has been hypothesized that the lower level of ACE2 and TMPRSS2 in pediatric upper airways epithelial cells limits viral infectivity in children ${ }^{17}$, although this has remained somewhat controversial ${ }^{18,19}$. In the present study, whilst there was a trend towards decreased ACE2 protein levels in pediatric NECs there was significant donor-to-donor variability that precluded statistical significance. We interpret these data as suggesting that ACE2 levels may contribute to, but are not the sole factor, in the increased resistance of children to SARS-CoV-2.

Despite donor-to-donor differences in ACE2 expression, we consistently observed a significant reduction in SARS-CoV-2 (QLD02) replication in pediatric NECs compared to NECs of adults. Given that the nasal epithelium is the first site of SARS-CoV-2 infection these data are consistent with the reduced number of SARS-CoV-2 infected children recorded in epidemiological studies ${ }^{43,44}$. There have been previous suggestions that nasopharyngeal SARSCoV-2 titers in children and adolescents are equivalent to those of adults ${ }^{45-47}$. However, reanalysis of the aforementioned studies has shown that young children ( $<10$ years old) did indeed have a significantly lower viral load ${ }^{48}$, or that the comparison was being performed between children in the first 2 days of symptoms and hospitalized adults with severe disease 49 or that the dataset included few children younger than 16 years ${ }^{50}$. Indeed, it is challenging to compare data from controlled experimental studies to data obtained from patient sampling, where it is difficult to control for time of sampling relative to the onset of infection. Rather, decreased viral replication in pediatric epithelial cells is consistent with experimental studies in ferrets where aged ferrets showed higher viral load and longer nasal virus shedding ${ }^{51}$.

Consistent with reduced SARS-CoV-2 replication in the nasal epithelium of children, pediatric epithelial cells had a more pronounced pro-inflammatory response (compared to adult cells) following a SARS-CoV-2 infection. In particular, a pronounced interferon response and the expression of interferon stimulated genes (ISGS) was higher in infected pediatric, compared to adult, NECs. Increased $I S G$ expression, and the subsequent anti-viral response may contribute to the reduced viral replication observed in pediatric cells. Importantly, unlike the lower respiratory tract, any resultant cell death or immunopathology in the upper respiratory tract is unlikely to lead to respiratory distress and therefore remains beneficial to the host ${ }^{24}$. These 
findings are consistent with those of Maughan et al, who analyzed transcriptional profile of airway (tracheobronchial) epithelium and observed upregulated type I and II IFNs associated genes in children ${ }^{23}$. Similarly, in the nasal fluid of children and adults presenting to the emergency department with SARS-CoV-2 there were significantly higher levels of IFN- $\alpha 2$ in the fluid derived from children. Increased interferon signaling was also recorded in the nasopharyngeal transcriptome of children compared to that of adults during early SARS-CoV2 infection ${ }^{18,22}$. The question remains as to why pediatric epithelial cells mount a stronger inflammatory and anti-viral response to SARS-CoV-2 compared to adult cells. This may represent an adaptation to the increased antigenic challenge observed in childhood. Alternatively, it is possible that increased antigenic exposure in childhood 'trains' nasal epithelium in children to mount a stronger pro-inflammatory response to any antigenic challenge. It is also possible that metabolic differences between pediatric and NECs (as potentially suggested by the different morphologies of the cells) could alter gene expression. It is also important to recognize that these data may not be applicable to all patient populations as previous studies of children and adults hospitalized with COVID-19 did not find an agedependent difference in the interferon response ${ }^{52}$.

A pronounced pro-inflammatory and anti-viral response in pediatric cells was not restricted to SARS-CoV-2 infection and a similar result was observed following influenza virus infection. However, influenza virus replicated equally as well in pediatric cells compared to adults. These data are consistent with the high sensitivity of children to influenza virus infection ${ }^{39}$. These data are also seemingly discordant with the increased interferon response of pediatric cells. However, SARS-CoV-2 is highly sensitive to interferon treatment, more so than influenza A virus ${ }^{53,54}$. Therefore, we speculate that whilst the differential interferon response between children and adults is sufficient to inhibit SARS-CoV-2 replication, it is not sufficient to inhibit influenza virus replication.

The growing dominance of SARS-CoV-2 VOCs has raised speculation that the epidemiology of SARS-CoV-2 infection has fundamentally changed. Namely, there have been suggestions children are more susceptible to VOCs compared to the original Wuhan isolate ${ }^{55}$. This is difficult to discern using epidemiological data alone, as data are confounded by the fact that unlike adults, young children are not routinely vaccinated. The data presented here would suggest that the pediatric epithelium still confers some protection against the replication of the Alpha variant. Whether this remains true of other VOCs (including the more recent Delta variant), and in a more complex in vivo situation, remains to be determined.

Finally, it is important to recognize the limitations of this study. Due to the difficulties associated with obtaining NECs from children only a limited number of donors could be used for this study. However, as donors were not selected according to susceptibility to respiratory 
viral infection, their responses should be broadly representative of healthy children. Furthermore, our data focused on the role of nasal epithelial cells in age-dependent differences in SARS-CoV-2 infection. However, there may be other mechanisms to explain the reduced susceptibility of children to SARS-CoV-2 infection that were not measured in the present study. For example, children and adolescents have much higher titers of preexisting antibodies to SARS-CoV-2 compared to adults ${ }^{16}$. This study is unable to ascertain if this plays a more significant role than the nasal epithelium in protecting children from infection in vivo. Finally, it is important to recognize that it is possible that the data presented herein were not the result of age differences between pediatric and adult NECs and were instead the result of another undefined factor that was also different between the two patient groups.

Despite these potential limitations, the data presented here strongly suggest that the nasal epithelium of children is distinct and that it may afford children some level of protection from SARS-CoV-2 infection.

\section{Author Contributions:}

The authors meet criteria for authorship as recommended by the International Committee of Medical Journal Editors, contributed to the manuscript and have given final approval for the version to be published. Y.Z. and K.R.S. wrote the manuscript. T.R.K, A.C.B., K.M.S. and K.R.S. designed the study, Y.Z., K.Y.C., A.Y., L.L, T.Y., A.A.K., C.J.S. and H.B.O. collected the data. Y.Z., K.Y.C., A.C.K., C.J.S., Y.X., D.M., A.K., M.J., G.B., F.A.M. and K.R.S. analyzed the data. Y.Z., A.C.K. M.J., G.B. and K.R.S. designed the figures. P.D.S. K.M.S. and K.R.S. recruited study participants. All authors approved the final manuscript.

\section{Acknowledgments}

We greatly thank the participants in the study and the members of the research team. We would also like to acknowledge health care providers and their families worldwide.

\section{Funding}

This work is supported by the Australia Research Council (Fellowship DE180100512 to K.R.S and Discovery Early Career Researcher Award DE190100565 to M.J.), The National Health and Medical Research Council (Project grant APP1139316 and Senior research Fellowship APP1155794 to F.A.M.), and Academy of Finland and COVID19 research donations (Grant 318434 to G.B.). 


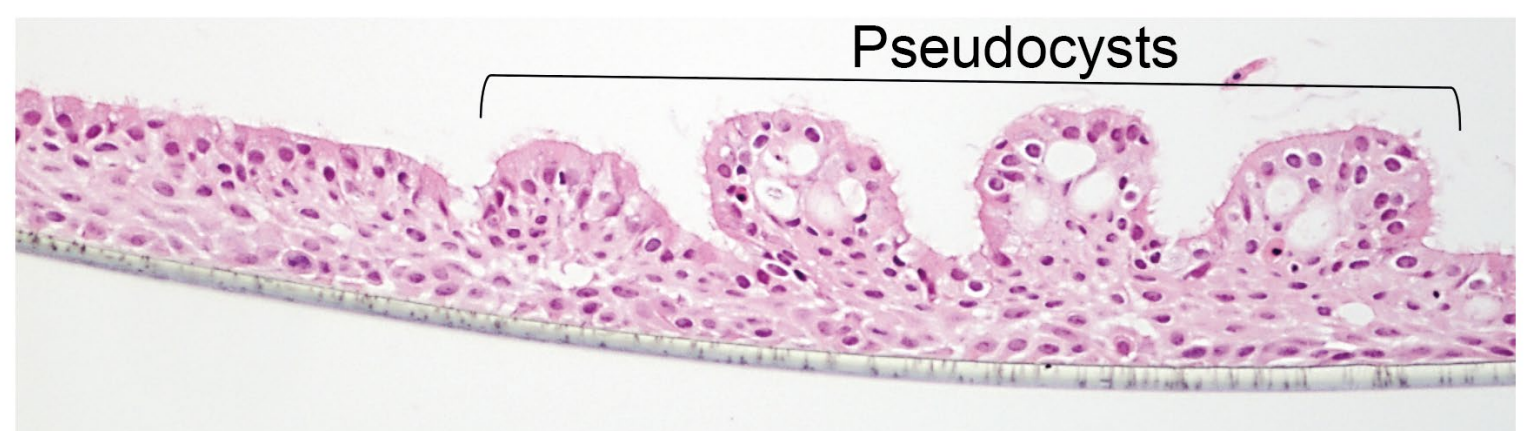

\section{Supplementary Figure 1: Pediatric nasal epithelial cells display pseudocysts.} Representative Hematoxylin \& Eosin-stained section of pediatric NECs culture differentiated at an air-liquid interface.

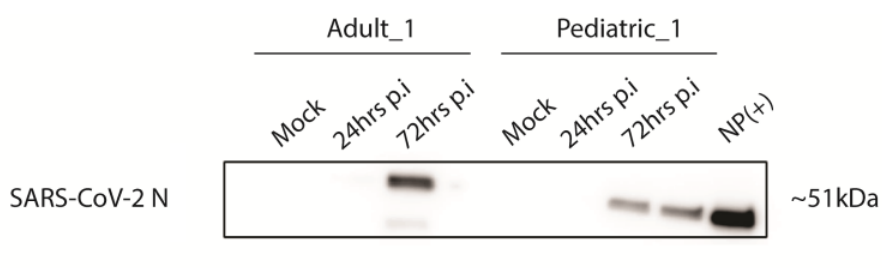

GAPDH
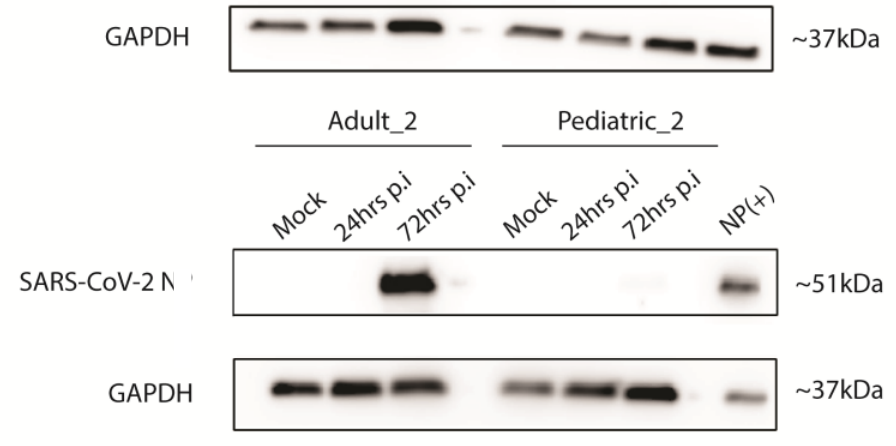

SARS-CoV-2 Orf7a

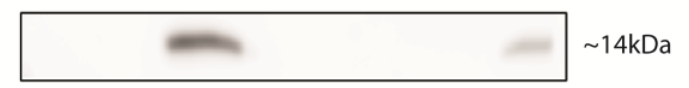

Adult_3

Pediatric_3

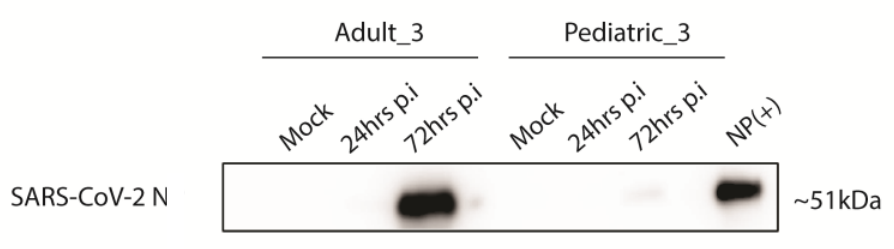

GAPDH

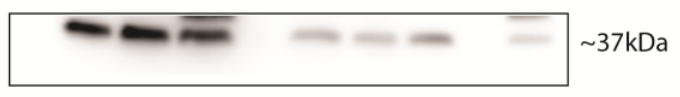

Supplementary Figure 2. Relative SARS-CoV-2 NP levels compared to GAPDH in pediatric and 
677

678

679

680

681

682

683

684

685

686

687

688

689

690

691

692

693

694

695

696

697

698

699

700

701

702

703

704

705

706

707

\section{REFERENCES}

1. Lu X, Zhang L, Du H, et al. SARS-CoV-2 Infection in Children. New England Journal of Medicine 2020; 382(17): 1663-5.

2. Lee P-I, Hu Y-L, Chen P-Y, Huang Y-C, Hsueh P-R. Are children less susceptible to COVID-19? J Microbiol Immunol Infect 2020; 53(3): 371-2.

3. Lanari M, Chiereghin A, Biserni GB, Rocca A, Re MC, Lazzarotto T. Children and SARS-CoV-2 infection: innocent bystanders...until proven otherwise. Clin Microbiol Infect 2020; 26(9): 1130-2.

4. Team CC-R. Coronavirus Disease 2019 in Children - United States, February 12-April 2, 2020. MMWR Morb Mortal Wkly Rep 2020; 69(14): 422-6.

5. Tagarro A, Epalza C, Santos M, et al. Screening and Severity of Coronavirus Disease 2019 (COVID19) in Children in Madrid, Spain. JAMA Pediatr 2021; 175(3): 316-7.

6. Kuchar E, Załęski A, Wronowski M, et al. Children were less frequently infected with SARS-CoV-2 than adults during 2020 COVID-19 pandemic in Warsaw, Poland. European Journal of Clinical Microbiology \& Infectious Diseases 2021; 40(3): 541-7.

7. Zhu Y, Bloxham CJ, Hulme KD, et al. A meta-analysis on the role of children in SARS-CoV-2 in household transmission clusters. Clin Infect Dis 2020: ciaa1825.

8. Goldstein E, Lipsitch M, Cevik M. On the Effect of Age on the Transmission of SARS-CoV-2 in Households, Schools, and the Community. The Journal of Infectious Diseases 2021; 223(3): 362-9.

9. Hua C-Z, Miao Z-P, Zheng J-S, et al. Epidemiological features and viral shedding in children with SARS-CoV-2 infection. Journal of Medical Virology 2020; 92(11): 2804-12.

10. Khateeb J, Li Y, Zhang H. Emerging SARS-CoV-2 variants of concern and potential intervention approaches. Critical Care 2021; 25(1): 244.

11. Nyberg T, Twohig KA, Harris RJ, et al. Risk of hospital admission for patients with SARS-CoV-2 variant B.1.1.7: cohort analysis. Bmj 2021; 373: n1412.

12. Somekh I, Sharabi A, Dory Y, Simões EAF, Somekh E. Intrafamilial Spread and Altered Symptomatology of SARS-CoV-2, During Predominant Circulation of Lineage B.1.1.7 Variant in Israel. Pediatr Infect Dis J 2021; 40(8): e310-e1.

13. Ladhani SN, Ireland G, Baawuah F, et al. Emergence of SARS-CoV-2 Alpha (B.1.1.7) variant, infection rates, antibody seroconversion and seroprevalence rates in secondary school students and staff: active prospective surveillance, December 2020 to March 2021, England. Journal of Infection 2021. 
14. Meyer M, Holfter A, Ruebsteck E, et al. The Alpha Variant (B.1.1.7) of SARS-CoV-2 in Children: First Experience from 3544 Nucleic Acid Amplification Tests in a Cohort of Children in Germany. Viruses 2021; 13(8).

15. Longini IM, Jr., Koopman JS, Monto AS, Fox JP. Estimating household and community transmission parameters for influenza. Am J Epidemiol 1982; 115(5): 736-51.

16. Ng KW, Faulkner N, Cornish GH, et al. Preexisting and de novo humoral immunity to SARS-CoV-2 in humans. Science 2020; 370(6522): 1339-43.

17. Bunyavanich S, Do A, Vicencio A. Nasal Gene Expression of Angiotensin-Converting Enzyme 2 in Children and Adults. JAMA 2020; 323(23): 2427-9.

18. Pierce CA, Sy S, Galen B, et al. Natural mucosal barriers and COVID-19 in children. JCI Insight 2021; 6(9).

19. Wark PAB, Pathinayake PS, Kaiko G, et al. ACE2 expression is elevated in airway epithelial cells from older and male healthy individuals but reduced in asthma. Respirology 2021: doi: 10.1111/resp.14003.

20. Hoffmann M, Kleine-Weber H, Schroeder S, et al. SARS-CoV-2 Cell Entry Depends on ACE2 and TMPRSS2 and Is Blocked by a Clinically Proven Protease Inhibitor. Cell 2020; 181(2): 271-80.e8.

21. Saheb Sharif-Askari N, Saheb Sharif-Askari F, Alabed M, et al. Airways Expression of SARS-CoV-2 Receptor, ACE2, and TMPRSS2 Is Lower in Children Than Adults and Increases with Smoking and COPD. Molecular Therapy - Methods \& Clinical Development 2020; 18: 1-6.

22. Loske J, Röhmel J, Lukassen S, et al. Pre-activated anti-viral innate immunity in the upper airways controls early SARS-CoV-2 infection in children. medRxiv 2021: 2021.06.24.21259087.

23. Maughan EF, Nigro E, Pennycuick A, et al. Cell-intrinsic differences between human airway epithelial cells from children and adults. bioRxiv 2020: 2020.04.20.027144.

24. Lucas $\mathrm{C}$, Wong $\mathrm{P}$, Klein J, et al. Longitudinal analyses reveal immunological misfiring in severe COVID-19. Nature 2020; 584(7821): 463-9.

25. Short KR, Kroeze EJBV, Fouchier RAM, Kuiken T. Pathogenesis of influenza-induced acute respiratory distress syndrome. The Lancet Infectious Diseases 2014; 14(1): 57-69.

26. Hou YJ, Okuda K, Edwards CE, et al. SARS-CoV-2 Reverse Genetics Reveals a Variable Infection Gradient in the Respiratory Tract. Cell 2020; 182(2): 429-46.e14.

27. Gordon DE, Jang GM, Bouhaddou M, et al. A SARS-CoV-2 protein interaction map reveals targets for drug repurposing. Nature 2020; 583(7816): 459-68. 
28. Short KR, Diavatopoulos DA, Reading PC, et al. Using bioluminescent imaging to investigate synergism between Streptococcus pneumoniae and influenza A virus in infant mice. J Vis Exp 2011; (50).

29. Siegers JY, Novakovic B, Hulme KD, et al. A High-Fat Diet Increases Influenza A Virus-Associated Cardiovascular Damage. The Journal of Infectious Diseases 2020; 222(5): 820-31.

30. S A. FastQC: a quality control tool for high throughput sequence data. Available online at: http://www.bioinformatics.babraham.ac.uk/projects/fastqc). 2020.

31. Ewels $P$, Magnusson $M$, Lundin S, Käller M. MultiQC: summarize analysis results for multiple tools and samples in a single report. Bioinformatics 2016; 32(19): 3047-8.

32. Patro R, Duggal G, Love MI, Irizarry RA, Kingsford C. Salmon provides fast and bias-aware quantification of transcript expression. Nat Methods 2017; 14(4): 417-9.

33. Love MI, Huber W, Anders S. Moderated estimation of fold change and dispersion for RNA-seq data with DESeq2. Genome Biol 2014; 15(12): 550.

34. Ritchie ME, Phipson $B, W u$, et al. limma powers differential expression analyses for RNAsequencing and microarray studies. Nucleic Acids Res 2015; 43(7): e47.

35. Law CW, Chen Y, Shi W, Smyth GK. voom: Precision weights unlock linear model analysis tools for RNA-seq read counts. Genome Biol 2014; 15(2): R29.

36. Young MD, Wakefield MJ, Smyth GK, Oshlack A. Gene ontology analysis for RNA-seq: accounting for selection bias. Genome Biology 2010; 11(2): R14.

37. Bardanzellu F, Fanos V. How could metabolomics change pediatric health? Italian Journal of Pediatrics 2020; 46(1): 37.

38. Yazicioglu T, Mühlfeld $C$, Autilio $C$, et al. Aging impairs alveolar epithelial type Il cell function in acute lung injury. Am J Physiol Lung Cell Mol Physiol 2020; 319(5): L755-169.

39. Kondrich J, Rosenthal M. Influenza in children. Curr Opin Pediatr 2017; 29(3): 297-302.

40. Lachassinne $\mathrm{E}$, de Pontual L, Caseris M, et al. SARS-CoV-2 transmission among children and staff in daycare centres during a nationwide lockdown in France: a cross-sectional, multicentre, seroprevalence study. Lancet Child Adolesc Health 2021.

41. Stringhini S, Wisniak A, Piumatti G, et al. Seroprevalence of anti-SARS-CoV-2 IgG antibodies in Geneva, Switzerland (SEROCoV-POP): a population-based study. Lancet 2020; 396(10247): 313-9. 
42. Tönshoff B, Müller B, Elling R, et al. Prevalence of SARS-CoV-2 Infection in Children and Their Parents in Southwest Germany. JAMA Pediatrics 2021.

43. Mehta NS, Mytton OT, Mullins EWS, et al. SARS-CoV-2 (COVID-19): What Do We Know About Children? A Systematic Review. Clin Infect Dis 2020; 71(9): 2469-79.

44. Zimmermann P, Curtis N. Why is COVID-19 less severe in children? A review of the proposed mechanisms underlying the age-related difference in severity of SARS-CoV-2 infections. Archives of Disease in Childhood 2020: archdischild-2020-320338.

45. Baggio S, L'Huillier AG, Yerly S, et al. SARS-CoV-2 viral load in the upper respiratory tract of children and adults with early acute COVID-19. Clin Infect Dis 2020.

46. Chung E, Chow EJ, Wilcox NC, et al. Comparison of Symptoms and RNA Levels in Children and Adults With SARS-CoV-2 Infection in the Community Setting. JAMA Pediatr 2021.

47. Jacot D, Greub G, Jaton K, Opota O. Viral load of SARS-CoV-2 across patients and compared to other respiratory viruses. Microbes Infect 2020; 22(10): 617-21.

48. Li X, Xu W, Dozier M, He Y, Kirolos A, Theodoratou $E$. The role of children in transmission of SARS-CoV-2: A rapid review. J Glob Health 2020; 10(1): 011101.

49. Yonker LM, Neilan AM, Bartsch Y, et al. Pediatric Severe Acute Respiratory Syndrome Coronavirus 2 (SARS-CoV-2): Clinical Presentation, Infectivity, and Immune Responses. J Pediatr 2020; 227: 45-52.e5.

50. Singanayagam A, Patel $M$, Charlett $A$, et al. Duration of infectiousness and correlation with RTPCR cycle threshold values in cases of COVID-19, England, January to May 2020. Euro Surveill 2020; 25(32).

51. Young-Il K, Kwang-Min Y, June-Young K, et al. Age-dependent pathogenic characteristics of SARS-CoV-2 infection in ferrets. Research Square 2021.

52. Koch CM, Prigge AD, Anekalla KR, et al. Immune response to SARS-CoV-2 in the nasal mucosa in children and adults. medRxiv 2021: 2021.01.26.21250269.

53. Blanco-Melo D, Nilsson-Payant BE, Liu W-C, et al. Imbalanced Host Response to SARS-CoV-2 Drives Development of COVID-19. Cell 2020; 181(5): 1036-45.e9.

54. Wu W, Metcalf JP. The Role of Type I IFNs in Influenza: Antiviral Superheroes or Immunopathogenic Villains? Journal of Innate Immunity 2020; 12(6): 437-47. 
bioRxiv preprint doi: https://doi.org/10.1101/2021.03.08.434300; this version posted August 24, 2021. The copyright holder for this preprint (which was not certified by peer review) is the author/funder. All rights reserved. No reuse allowed without permission.

797

798

799 Virulence 2021; 12(1): 2013-6. 55. van Oosterhout C, Stephenson JF, Weimer B, Ly H, Hall N, Tyler KM. COVID-19 adaptive evolution during the pandemic - Implications of new SARS-CoV-2 variants on public health policies. 